\title{
Understanding Cygnus X-3 Through Multi-Wavelength Studies
}

\section{Michael McCollough*t}

Smithsonian Astrophysical Observatory, U.S.A.

E-mail: mmccollough@head. cfa.harvard.edu

\section{Karri Koljonen}

Aalto University Metsähovi Radio Observatory, Finland

E-mail: karriekurp.hut.fi

\section{Diana Hannikainen}

Aalto University Metsähovi Radio Observatory/Finnish Centre for Astronomy with ESO, Finland

E-mail: diana@kurp.hut.fi

\section{Guy Pooley}

Astrophysics Group, Cavendish Laboratory, U.K.

E-mail: guy@mrao.cam.ac.uk

\section{Sergei A. Trushkin}

Special Astrophysical Observatory RAS, Russia

E-mail: sergei .trushkin@gmail.com

\section{Danny Steeghs}

University of Warwick, U.K.

E-mail: D.T.H.Steeghs@warwick.ac.uk

\section{Marco Tavani}

INAF-IASF, Italy

E-mail: marco.tavani@iasf-roma.inaf.it

\section{Robert Droulans}

CESR/CNRS - Université de Toulouse, France

E-mail: Robert.Droulansecesr.fr

Cygnus X-3 is a unique microquasar which shows X-ray state changes, strong radio emission, and relativistic jets. It is also an unusual X-ray binary with the mass-donating companion being a high mass star Wolf-Rayet but the orbital modulation (as inferred from X-ray emission) is only 4.8 hours, a value more common in low-mass systems. It has recently been shown by AGILE and Fermi that Cygnus X-3, is a transient gamma-ray source (>100 MeV).

To understand the environment, nature, and behavior of Cygnus X-3 multi-wavelength observations are necessary. In this proceedings we present the results achieved so far from multiwavelength campaigns.

8th INTEGRAL Workshop "The Restless Gamma-ray Universe"

September 27-30 2010

Dublin Castle, Dublin, Ireland 


\section{Introduction}

Cygnus X-3 is a unique microquasar composed of a compact object and a Wolf-Rayet star [1]. It has a strong 4.8 hour orbital modulation and is known to produce relativistic jets [2,3]. It also exhibits relatively strong radio emission most of the time ( $\sim 100 \mathrm{mJy})$ [4]. AGILE [5] and Fermi [6] have recently shown that Cygnus X-3 is a transient gamma-ray source ( $>100 \mathrm{MeV})$. Flaring gamma-ray emission appears to be connected with major radio flares and their associated X-ray states that precede the major radio flares [5, 7].

Studies $[4,8]$ in the radio and hard X-ray (HXR) have found four states: quiescent, minor flaring, quenched and major flaring. Table 1 shows how these states are correlated with the simultaneous X-ray observations. Throughout all states of activity the HXR and soft X-ray (SXR) show an anti-correlation or spectral pivoting around $10 \mathrm{keV}$ [9].

Additional studies have shown that the X-ray emission can be broken down into additional states. A study of the X-ray and radio behavior arrive at six states (quiescent, minor flaring, suppressed, post-flare, major flaring, and quenched) [10]. A recent study which incorporated X-ray spectral hardness (shape of the spectrum) in addition to the X-ray and radio arrive at three major states, Quiescent, Flaring, and Hypersoft, with several substates [11]. The Hypersoft is a new state which has important ties to the quenched state, gamma-ray emission, and jet production.

\section{Results}

The multi-wavelength campaigns (2006/2010) initiated to study the environment, nature and behavior of Cygnus X-3 included data from space-based (INTEGRAL, AGILE, Chandra, Swift, RXTE, and Fermi), ground-based radio (Ryle, RATAN-600, Metsähovia, VLBA, VLA, and WSRT), and Infrared (PAIRITEL, Calar Alto) observatories. Some of the initial results of these campaigns are presented below.

\subsection{Multi-Wavelength Look at a Major Radio Flare}

INTEGRAL Target of Opportunity Observations (TOOs) allowed a detailed look at Cygnus X-3's X-ray spectrum near the peak of major radio flares. These included observations within 0.25 days, 2.5 days, and 3.5 days of the flare peak in the radio. In addition to INTEGRAL TOOs, observations were made with RXTE, Swift, and ground-based radio and infrared observations (see $[12,13])$. This allowed us to assemble Cygnus X-3 SEDs during these major radio flares.

Initial fits with physical models such as Eqpair [14] and BELM [15] show: (a) Radio Spectrum: At the peak of the radio flare the radio spectrum has become optically thin; (b) Absorption: In all of the observations there is strong absorption from the infrared to the SXR; (c) Model Components: To model the data it was necessary to have a disk component, a compact corona, and a diluted jet. Most of the radio emission arises from outside of the core region; and (d) Optical Depth: As the flare evolves the optical depth of the core of the system decreases.

Some additional major findings from these campaigns were:

\footnotetext{
*Speaker.

${ }^{\dagger}$ This work is supported by NASA contract NAS8-03060.
} 

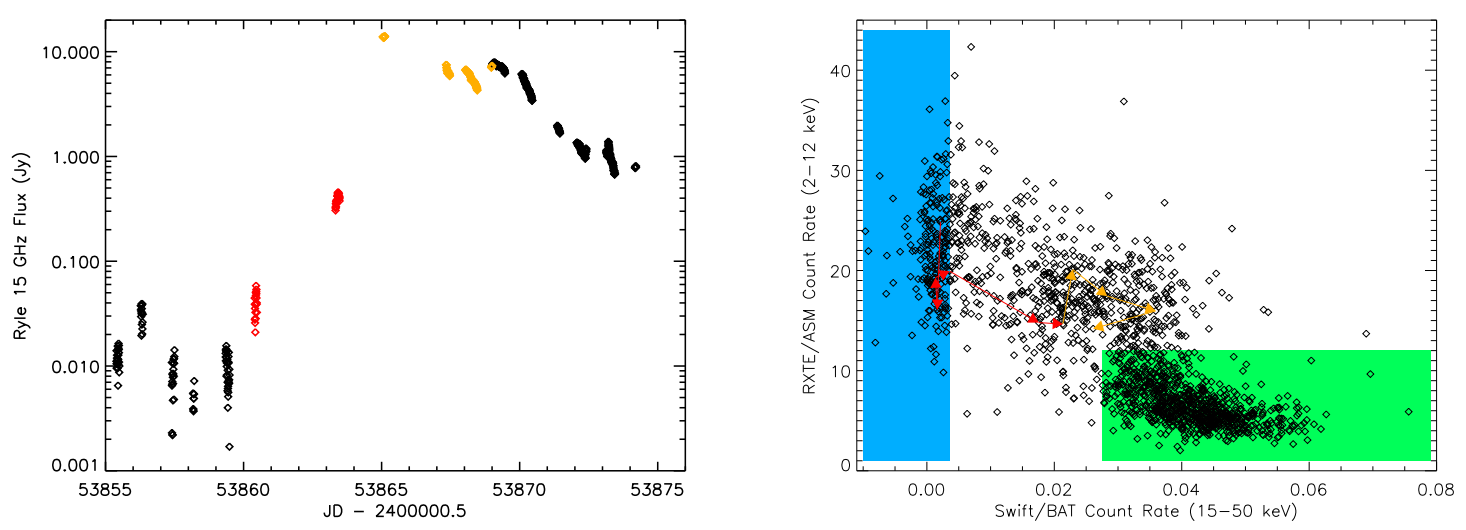

Figure 1: left: Radio light curve for the first major radio flare observed by INTEGRAL. Note the color coding for before the peak (red) and after (orange). right: A HXR vs. SXR plot for Cygnus X-3. The path followed during the major radio flare are shown with red before the peak and orange after. The blue represents the quenched state and the green the quiescent state.

Quenched State Trigger: For all of the major radio flares examined it has been found that Cygnus X-3 must pass through the quenched state $[10,11]$. Specifically it appears that Cygnus X-3 passing through the Hypersoft state may be crucial for the formation of major radio flares [11]. In order to determine if Cygnus X-3 is in a quenched state an examination of both the SXR and HXR is necessary in addition to the radio. Quenching in the radio may not always be observed due to the radio emission from decaying earlier flares (post-flare state [10]).

Correlation with the HXR: When Cygnus X-3 is in a flaring state the HXR and radio are correlated with each other. The HXR appears to lag the radio in the 2006 major flare between three to five days (see Fig. 1). During the flaring state the HXR values are in general below those seen during quiescence.

X-Ray Spectral Evolution: The X-ray spectrum of Cygnus X-3 consistently evolves from a soft spectrum (Hypersoft) at the beginning of the flare to become progressively harder as the flare peaks and decays (see Fig. 1). This is due to the presence of a HXR tail that initially increases in intensity and then becomes fainter and harder as the flare decays.

Low Frequency QPOs: A recent discovery is that low frequency QPOs (LFQPOs), first reported by [16], are common and associated with the major radio flares [17]. The LFQPOs are found to follow a major radio flare and are observed only in the orbital phase range of $0.2-0.7$ [17].

\subsection{Gamma-Ray Emission}

Cygnus X-3 has been detected by various space missions out to energies of $\sim 200-300 \mathrm{keV}$. Throughout the 1970s and 1980s there were reported detections of Cygnus X-3 in the MeV to $\mathrm{TeV}$ energy ranges. But none of these detections were confirmed or found reproducible. Recent observations by AGILE and Fermi have shown that the Cygnus region is a very complicated area at gamma-ray energies. Cygnus X-3 has now been shown by both AGILE [5] and Fermi [6] to be a gamma-ray source $(>100 \mathrm{MeV})$. 


\begin{tabular}{|c|c|c|c|c|c|}
\hline State & Radio Level & SXR & HXR & Radio - SXR & Radio - HXR \\
\hline quiescent & $\sim 60-100 \mathrm{mJy}$ & low & high & + & - \\
\hline minor flaring & $<1$ Jy & $\uparrow$ & $\downarrow$ & $*$ & $*$ \\
\hline quenched & $\sim 1-15 \mathrm{mJy}$ & high & low & - & + \\
\hline major flaring & $\sim 1-20 \mathrm{Jy}$ & $\downarrow$ & $\uparrow$ & - & + \\
\hline
\end{tabular}

Table 1: For each of Cygnus X-3's states [4, 8] is given the radio level, SXR and HXR behavior (high, low, rising $\uparrow$ or falling $\downarrow$ ) and correlations between the radio and the SXR and HXR. For the correlations + is a correlation and - is an anti-correlation. The * represents where no correlation has been found.
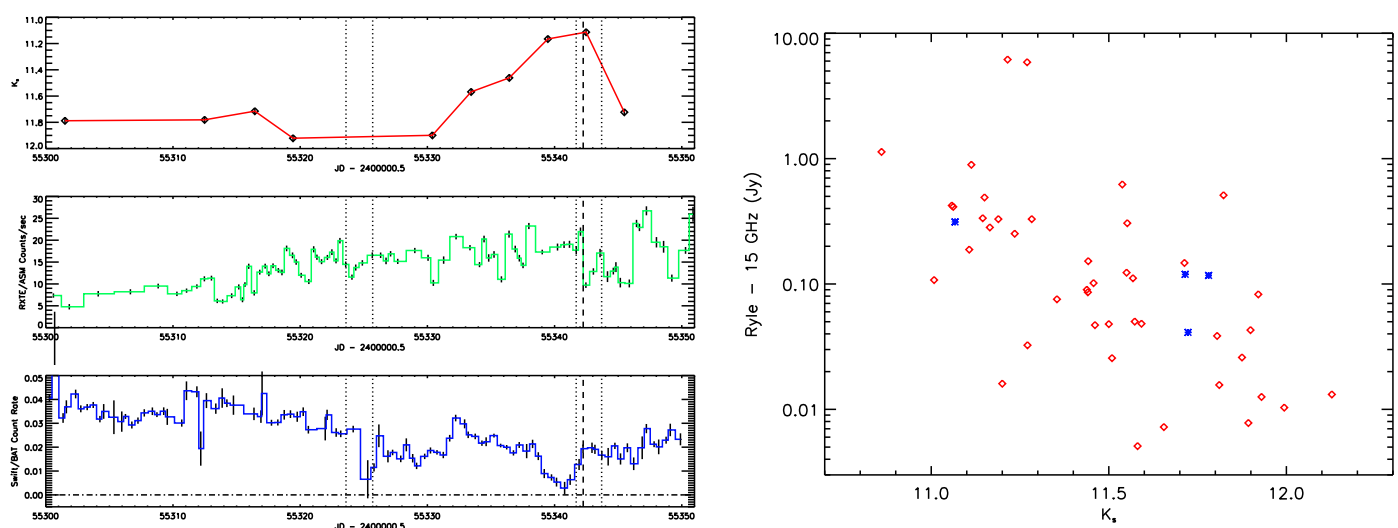

Figure 2: left: Lightcurves of the infrared (red), SXR (green) and HXR (blue) during times of gamma-ray emission (dotted vertical lines) and a $1 \mathrm{Jy}$ radio flare (dashed vertical line). right: Infrared magnitude $\left(\mathrm{K}_{\mathrm{S}}\right)$ vs. radio flux density $(15 \mathrm{GHz})$. Note the logarithmic y-axis. The red points were taken during X-ray flaring states and the blue points during X-ray quiescence.

AGILE detected gamma-ray emission during periods of HXR quenching and very high SXR activity (typical Cygnus X-3 quenched state). The gamma-ray spectrum between $100 \mathrm{MeV}$ and 3 $\mathrm{GeV}$ is well described by a power-law spectrum with a photon index of $1.8 \pm 0.2$. The gamma-ray emission appear to be correlated with the recently identified Hypersoft state [11].

Fermi found a $29 \sigma$ source consistent with Cygnus X-3. Fermi also found that using only data taken during periods of flaring activity, the gamma-ray emission exhibited the 4.8 hour orbital modulation associated with Cygnus X-3. Although it was found to be shifted by $0.3-0.4$ in phase relative what is observed at other wavelengths.

In May 2010 AGILE and Fermi both detected renewed gamma-ray emission from Cygnus X-3 [20]. In both episodes of activity the gamma-ray emission was associated with very brief periods of HXR quenching. In one case the HXR quenching lasted only $\sim 0.5$ day and in the other for a 1 day. In the last period of activity a $1 \mathrm{Jy}$ radio flare $(15 \mathrm{GHz})$ was observed during the period of gamma-ray emission (see Fig. 2).

Various models have been proposed to explain the gamma-ray emission in terms of leptonic [18] or hadronic processes [19]. 

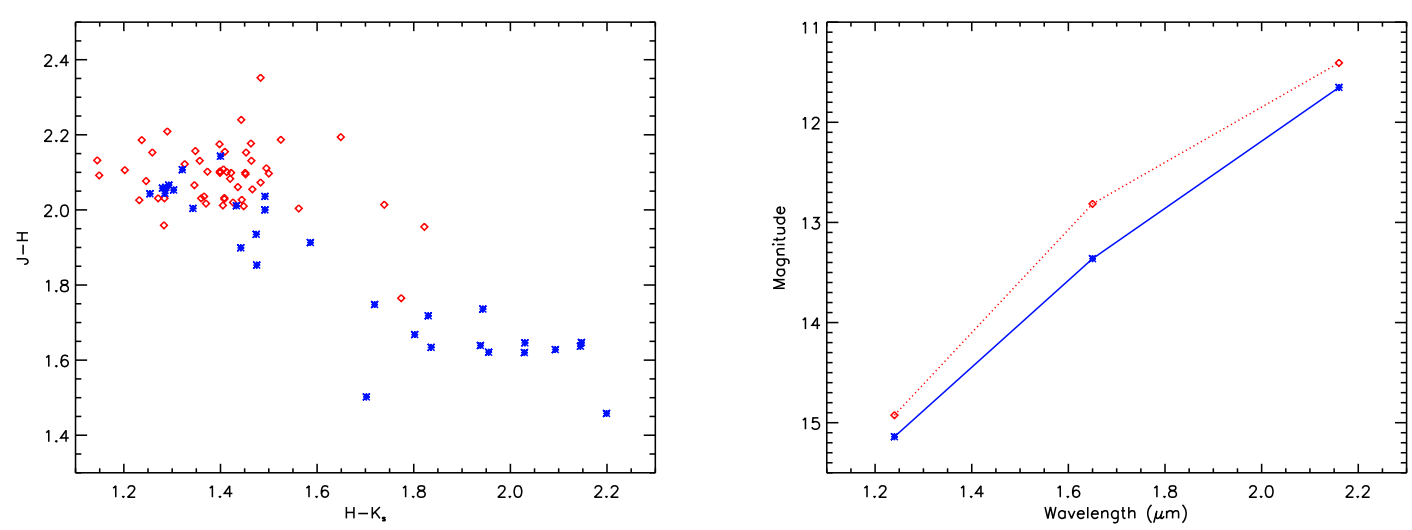

Figure 3: left: An infrared color-color plot. Note the correlation between the colors which is heavily driven by the state changes. The red represent observations during a flaring state and blue during a quiescent state. right: The average spectrum for the flaring (red) state and quiescent (blue). The wavelength is that of the band center.

\subsection{Infrared Emission}

Because of the heavy absorption, due to interstellar and intrinsic material, Cygnus X-3 is not observable in the optical and UV. For this reason the infrared is an important link between the radio and X-ray.

It was from infrared spectra that the mass-donating companion of Cygnus X-3 was identified to be a Wolf-Rayet star [1]. Infrared observations show the $4.8 \mathrm{hr}$ orbital modulation similar to that seen in the X-ray, although it is not as strong (10\% as compared to $50 \%$ in the X-ray) [21]. There have been observations for which the infrared modulation has not been present [22]. Additionally there have been observations of short intense (factor of 2) flares imposed on top of the orbital modulation [21].

To improve our coverage in the infrared as part of our multi-wavelength campaign we are observing in the near infrared using the PAIRITEL telescope. PAIRITEL is the $1.3 \mathrm{~m}$ automated telescope used for the 2MASS survey. The final image mosaic created from an observation yields a 10 arcminute field with arcsecond resolution. For Cygnus $\mathrm{X}-3$ simultaneous $\left(\mathrm{J}, \mathrm{H}\right.$, and $\left.\mathrm{K}_{\mathrm{s}}\right)$ observations are achieved with a net exposure of $\sim 600 \mathrm{~s}$.

Cygnus X-3 Infrared Spectral Changes: An initial examination of the infrared shows that as Cygnus X-3 changes state (quiescent to flaring), the infrared spectrum changes. An infrared color - color plot $\left(\mathrm{H}-\mathrm{K}_{\mathrm{s}}\right.$ vs.J $\left.-\mathrm{H}\right)$ is shown in Fig. 3 which shows a clear statistically significant correlation (see Table 2). In Fig. 3 the average infrared spectrum is shown during quiescent (blue) and flaring (red) states. While the flux in all bands rises in the flaring state it is important to note the $\mathrm{H}$ band $(1.51-1.78 \mu \mathrm{m})$ experiences a noticeably larger increase. This likely represents an increase in one of the emission components that make up the infrared emission.

SXR - HXR - Infrared Relationships: A comparison between the infrared and the SXR/HXR show a clear correlation (SXR) and anti-correlation (HXR) with the infrared flux. Fig. 4 shows both of these relations with the $\mathrm{H}$ band (also see Table 2). As the SXR increases the infrared flux increases and the HXR flux decreases. These correlations appear to be almost completely driven 

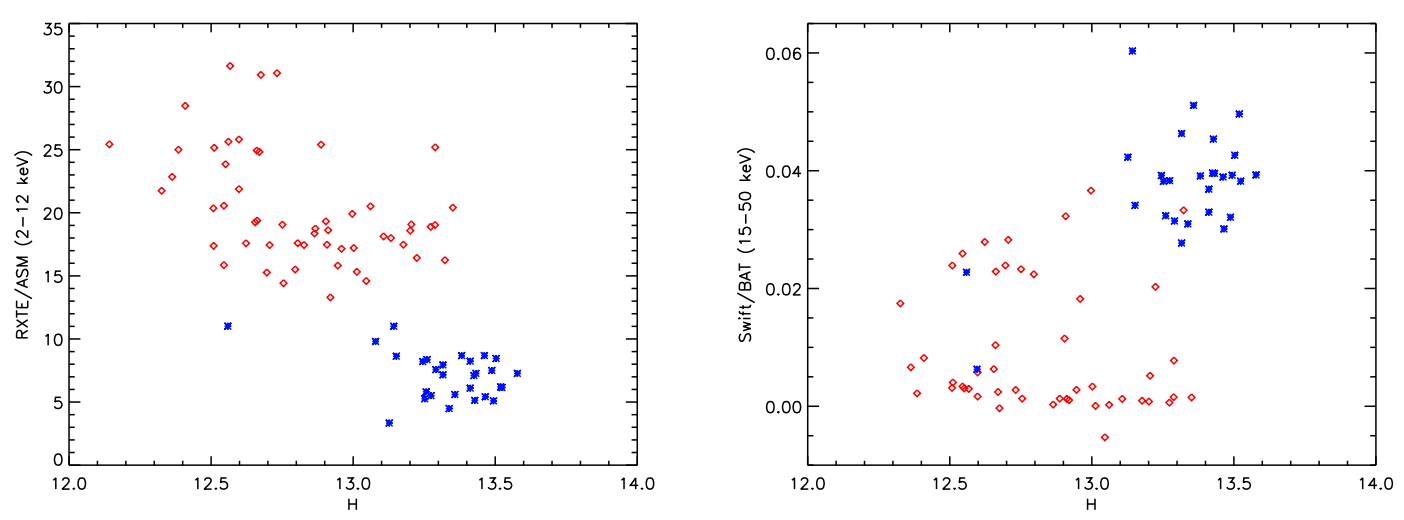

Figure 4: left: Plot of the $\mathrm{H}$ band magnitude vs. SXR. Red are flaring state observations and the blue are quiescent. right: Plot of the $\mathrm{H}$ band magnitude vs. HXR. Red are flaring state observations and the blue are quiescent.

\begin{tabular}{|c|c|c|c|}
\hline Band/Color & Band/Color & $\mathrm{r}_{\mathrm{s}}$ & $\sigma$ \\
\hline $\mathrm{H}-\mathrm{K}_{\mathrm{s}}$ & $\mathrm{J}-\mathrm{H}$ & -0.519 & $3 \times 10^{-7}$ \\
\hline $\mathrm{H}$ & $\mathrm{SXR}$ & -0.738 & $1 \times 10^{-15}$ \\
\hline $\mathrm{H}$ & $\mathrm{HXR}$ & 0.542 & $4 \times 10^{-7}$ \\
\hline $\mathrm{K}_{\mathrm{s}}$ & Radio & -0.640 & $1 \times 10^{-5}$ \\
\hline $\mathrm{H}$ & Radio & -0.558 & $3 \times 10^{-4}$ \\
\hline $\mathrm{J}$ & Radio & -0.513 & $1 \times 10^{-3}$ \\
\hline
\end{tabular}

Table 2: Infrared correlations: For different bands/colors Spearman rank-order correlation test was preformed. For each case the $r_{s}$ is the Spearman rank coefficient and $\sigma$ is significance (probability of no correlation) are given.

by the state change.

Infrared - Radio Correlation: Infrared observations were also matched with radio observations (maximum separation in time of $<$ day). Testing relations between the various infrared bands and the radio show a correlation of the radio flux density with the $\mathrm{K}_{\mathrm{s}}$ (see Fig. 2), a weak correlation with the $\mathrm{H}$ band, and a marginal correlation with the $\mathrm{J}$ band (see Table 2). One implication of this is that the synchrotron emission responsible for the radio emission extends down into the infrared with its strongest contribution in the longer wavelength band.

Infrared-Gamma-Ray Relationship: During the 2010 period of gamma-ray activity, infrared monitoring was also in place. In Fig. 2 are the infrared observations, SXR daily observations, and the HXR semi-daily observations during this time period. Labeled are periods of gamma-ray emission and the time of a $1 \mathrm{Jy}$ radio flare. Strikingly, the second period of gamma-ray emission, for which there is associated a radio flare, the infrared shows a direct response. The infrared starts to increase in the $\sim 14$ days period prior to the radio flare. Right after the peak of the radio flare the infrared shows a sudden decrease. There appear to be direct relationships between periods of gamma-ray emission, radio flares and infrared in Cygnus X-3. 


\section{Summary}

Through multi-wavelength campaigns of Cygnus X-3 important insights are being gained. Cygnus X-3 has been shown to be one of the few XRBs to produce gamma-ray emission. This emission is coupled to state behavior and major radio flares. The infrared is providing an important bridge between the radio and the X-ray. These studies are allowing us to probe some of the most extreme environments in nature: high mass flow in a strong gravitational field.

\section{References}

[1] van Kerkwijk, M. H. et al. 1992, Nature, 355, 703

[2] Parsignault, D. R., et al. 1972, Nature, 239, 123

[3] Mioduszewski, A. J. et al. 2001, ApJ, 553, 766

[4] Waltman, E. B. et al. 1994, AJ, 108, 179

[5] Tavani, M. et al. 2009, Nature, 462, 620

[6] Fermi LAT Collaboration et al. 2009, Science, 326, 1512

[7] McCollough, M. L., Koljonen, K. I. I., \& Hannikainen, D. C. 2009, “7th AGILE Meeting: The Bright Gamma-Ray Sky”, http://arxiv.org/pdf/1003.4278v1

[8] McCollough, M. L., et al., 1999, ApJ, 517, 951

[9] McCollough, M. L. et al. 1997, 4th Compton Symposium, 813

[10] Szostek, A., Zdziarski, A. A. \& McCollough, M. L. 2008,MNRAS, 388, 1001

[11] Koljonen, K. I. I. , et al. 2010, MNRAS, 406, 307

[12] McCollough, M. L., Koljonen, K. I. I. \& Hannikainen, D. C. 2008, “Proceedings of the 7th INTEGRAL Workshop”, http://pos.sissa.it/archive/conferences/067/090/Integral08_090.pdf

[13] Koljonen, K. I. I. \& McCollough, M. L. et al. 2008, “Proceedings of the 7th INTEGRAL Workshop”, http://pos.sissa.it/archive/conferences/067/075/Integral08_075.pdf

[14] Coppi, P. S., 1992 MNRAS, 258, 657

[15] Belmont, R., Malzac, J., \& Marcowich, A. 2008, A\&A, 491, 617

[16] van der Klis \& Jansen, F. A. 1985, Nature, 313, 768

[17] Koljonen, K. I. I. , et al., (in prep.)

[18] Dubus, G., Cerutti, B. \& Henri, G. 2010, MNRAS, 404, L55

[19] Romero, G. E. et al. 2003, $A \& A, \mathbf{4 1 0}, \mathrm{L} 1$

[20] Bulgarelli, A. et al. 2010, A\&A, (submitted)

[21] Mason, K. O., Cordova, F. A. \& White, N. E. 1986, ApJ, 309, 700

[22] Becklin, E. E. et al. 1974, ApJ, 192, 119 